\title{
Fabrication of Graphene Based Electrothermal Cantilever Actuator
}

\author{
Yilong Zhou, Hengchang Bi, Xiao Xie, Litao Sun* \\ SEU-FEI Nano-Pico Center, Key Laboratory of MEMS of Ministry of Education, Southeast University, Nanjing, China \\ *Corresponding Author: Litao Sun, $\underline{\text { slt } @ \text { seu.edu.cn }}$
}

\begin{abstract}
A novel actuator has been fabricated using graphene and graphene oxide composite paper as building blocks. The two materials have nearly equal Young's modulus, however a large displacement was observed during the electromechanically driven operation, which was attributed to great difference in coefficient of thermal expansion of graphene paper and graphene oxide paper.
\end{abstract}

Keywords- actuators; graphene; graphene oxide; bilayer

\section{INTRODUCTION}

"Paper-like" materials composed of graphene platelets produced by vacuum filtration of graphene suspensions exhibit good mechanical properties, with very high hardness of about $217 \mathrm{~kg} \mathrm{f} / \mathrm{mm}^{2}$ and yielding strength of about $6.4 \mathrm{TPa}$ [1]. In addition, graphene papers demonstrate outstanding bending rigidity and high elastic modulus under bending. These superior mechanical properties should render graphene paper an excellent material for actuators. Graphite oxide (GO) is a layered material consisting of hydrophilic oxygenated graphene sheets (graphene oxide sheets) bearing oxygen functional groups on their basal planes and edges [2]. Graphene oxide paper-like materials which are yielded through directed-flow assembly method possess a unique layered structure in which individual compliant graphene oxide sheets are interlocked or tiled together. It is this unique structure that endow graphene oxide papers with good mechanical properties including a modulus of about $40 \mathrm{GPa}$ and a fracture strength of about $130 \mathrm{MPa}$ [3].

Actuators, which can convert electric energy directly to mechanical energy, have attracted much attention. Since electromechanical properties of carbon nanotubes have been first reported, the related application acquired extensive attraction including nanopliers [4-5] and nanoswitches [6-9]. The use of two different building blocks to fabricate asymmetric materials has been proven a useful approach for producing mechanical actuators. Zhu et al. proposed a novel graphene-on-organic film fabrication method that was compatible with a batch microfabrication process. This method can be used for electromechanically driven microactuators which demonstrated a large displacement with rapid response while maintaining the low power consumption [10]. In addition, macroactuators based on carbon nanotubes /graphene oxide heterostructural papers have been reported by Park et al. Its actuation depended on variation of humidity and/or temperature [11]. By now, there have been no reports on actuators based on all-graphene sheets as building blocks.
Both graphene papers and graphene oxide papers have similar layered structures, which provide structural compatibility for a stable interface between these two layers. Moreover, based on the fact that both of graphene paper and graphene oxide paper have close Young's modulus (40 GPa) but exist huge difference of coefficient of thermal expansion [2, 12], the heterostructure composed of graphene paper and graphene oxide paper can be an ideal candidate for fabricating miniaturized actuators. These two different raw materials endow actuators with excellent mechanical properties.

\section{FABRICATION OF ACTUATORS BASED ON GRAPHENE/GRAPHENE OXIDE PAPERS}

\section{A. Preparation of graphite oxide}

GO was prepared by a modified Hummers method [13]. While maintaining agitation, expandable graphite powder (2 g) and sodium nitrate $(1 \mathrm{~g})$ were mixed in sulfuric acid $(46 \mathrm{ml}, 98$ $\mathrm{wt} \%)$ in an ice bath, and potassium permanganate $(6 \mathrm{~g})$ was slowly added to prevent the temperature from exceeding $20^{\circ} \mathrm{C}$. The reaction was kept at $35 \pm 1^{\circ} \mathrm{C}$ for $8 \mathrm{~h}$ with gas release, and then deionized water $(92 \mathrm{ml})$ was gradually added, bringing about violent effervescence. The temperature of the water bath was increase to $98{ }^{\circ} \mathrm{C}$ and the reaction was maintained for 15 min in order to increase the oxidation degree of the GO product. The resultant bright-yellow suspension was diluted with deionized $(280 \mathrm{ml})$ water and further treated with a $\mathrm{H}_{2} \mathrm{O}_{2}$ solution $(6 \mathrm{ml}, 30 \%)$. The product was separated by centrifugation, washed with $5 \%$ chlorhydric acid $(\mathrm{HCl})$ solution for 7 times until sulphate could not be detected with $\mathrm{BaCl}_{2}$, then also washed 7 times with distilled water aiming at removing chloride ions. Finally it was dried in an air oven at $60{ }^{\circ} \mathrm{C}$ overnight.

\section{B. Preparation of graphene}

The as-obtained graphite oxide ( $25 \mathrm{mg}$ ) was dispersed in DI water $(70 \mathrm{ml})$ and then exfoliated to generate graphene oxide nanosheets by ultrasonic wave. While maintaining agitation, hydrazine monohydrate $(0.35 \mathrm{ml}$, as reducing agent $)$ and ammonia solution (1.04 $\mathrm{ml}$, for adjusting $\mathrm{pH}$ value) were added. The temperature of the mixture solution was kept at $95{ }^{\circ} \mathrm{C}$ for $1 \mathrm{~h}$ in an oil bath, over which the color of the solution gradually changed to dark black as dispersed graphene nanosheets were formed. The dispersion was further centrifuged for $30 \mathrm{~min}$ at $3000 \mathrm{rpm}$ to remove a small amount 
of precipitates. The supernatant of the graphene nanosheets dispersion can be stored in vials for a long time.

\section{Fabrication of actuators}

Bilayer paper samples were successfully fabricated by sequential filtration of graphene dispersions and then graphene oxide suspension (Fig. 1a). Both surfaces of the circular bilayer paper were flat. The filtration sequence between graphene dispersion and grapene oxide suspension is determined by the hydrophobic nature of graphene sheets and hydrophilic nature of graphene oxide sheets. Reversing the sequential filtration, pre-filtrated graphene oxide layer would disperse into water more or less, which inevitably obscured a clear boundary between graphene layer and graphene oxide layer and had a bad effect on the surface of the graphene oxide layer. It was convenient that the thickness of each paper can be controlled by adjusting the concentration and volume of graphene and graphene oxide dispersions, respectively. The as-prepared bilayer paper was cut into a series of U-shaped beams using a sharp razor blade (Fig. 1b). In addition, copper wires were joined onto copper foils through electric welding. Then copper foils were conglutinated to glass substrates followed by conglutinating U-shaped beams to copper foils with conductive adhesive.

\section{RESULTS AND DISCUSSIONS}

Scanning electron microscopy (SEM) was adopted to characterize the surface and cross-section of graphene/graphene oxide paper (Fig. 2). It was clearly seen that, many great wrinkles were formed on the surface of the graphene oxide paper (Fig. 2a), which made graphene oxide paper possess rough surface. On the contrary, the surface of graphene paper looks relative smooth (Fig. 2b). Two main reasons were responsible for the above results. On the one hand, abundant functional groups, e.g. carboxyl groups, hydroxyl groups, etc., adhered to graphene oxide sheets stopped graphene oxide sheets from integrating compactly. On the other hand, the surface of filter paper exerted great compacts on the surface of graphene layer. In addition, the insets in Fig. 2 a demonstrated a dark brown and electrically
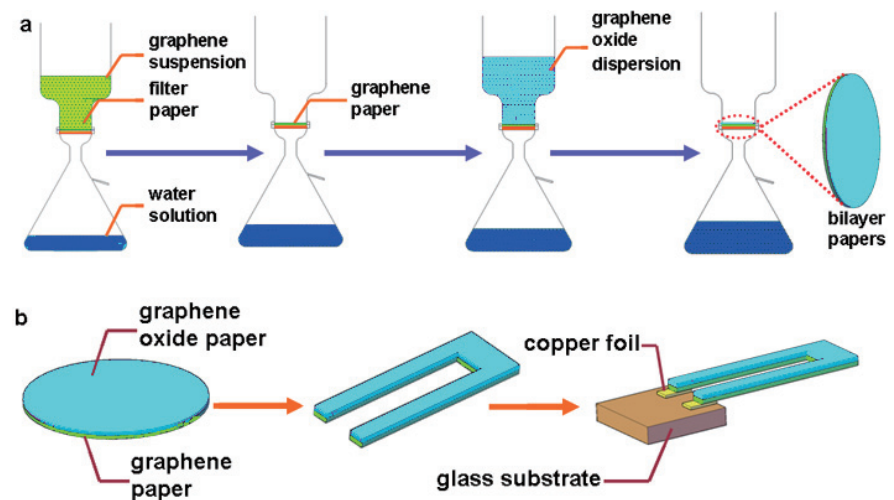

Fig. 1. Procedure of fabrication of actuator. Bilayer paper was produced though vacuum filtration of graphene dispersion and graphene oxide suspension in sequence, followed by being fabricated into actuator using a sharp razor blade.

insulating graphene oxide paper. On the contrary, the surface of graphene layer (insets in Fig. 2b) was black, shiny (metallic luster), and electrically conductive. The thickness and cross-

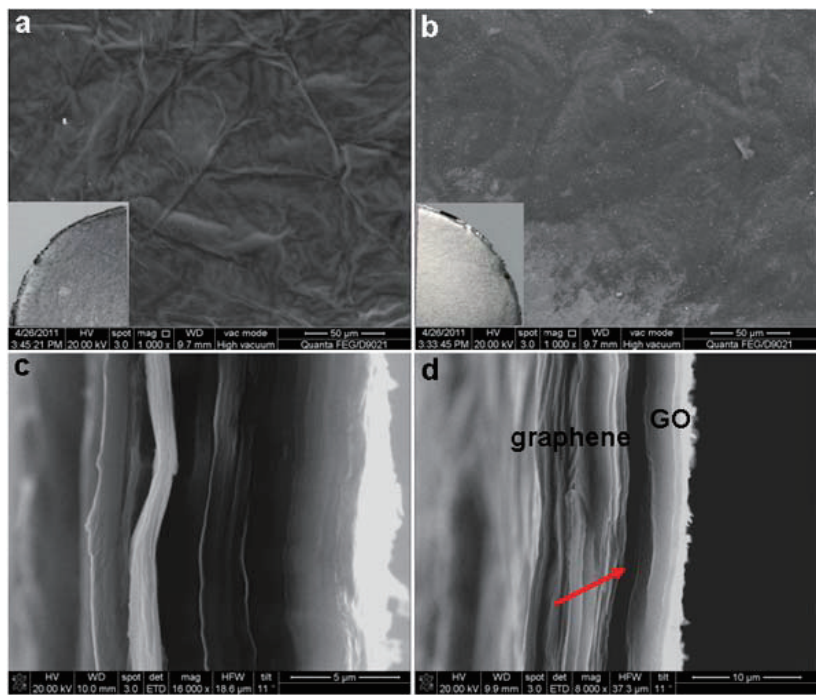

Fig. 2. The surface and cross-section morphology of bilayer papers. a, the surface of graphene oxide paper. b, the surface of graphene paper. c, the crosssection morphology of bilayer paper indicated compact combination. $\mathrm{d}$, the cross-section morphology of bilayer paper treated with liquid nitrogen.

section morphology of the bilayer paper can be acquired through SEM. It was hard to make a distinction between graphene paper and graphene oxide paper (Fig. 2c). The cross sections of each layer in the bilayer paper samples demonstrated the same morphology as the individually prepared graphene oxide [3] and graphene papers [1]. The bilayer paper did not delaminate at the macroscale, even at nanoscale, which was very beneficial for the operation of the actuators. In order to measure the thickness of graphene paper and graphene oxide paper individually, Liquid nitrogen was used to treat the bilayer paper. Falling off suddenly in temperature caused the separation between graphene paper and graphene oxide paper indicated by a red arrow in Fig. 2d, which was in favor of the measurements of their respective

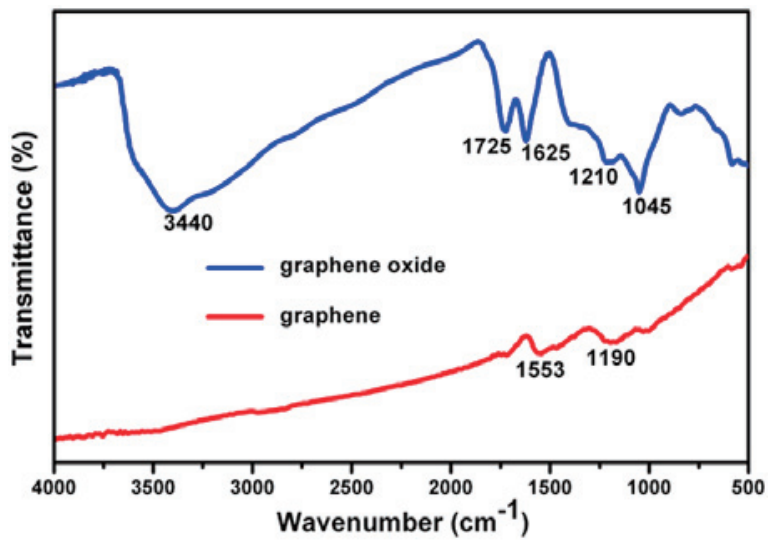

Fig. 3. IR spectra of graphene and graphene oxide. In the IR spectra of graphene oxide, the absorptions at 3440 and $1725 \mathrm{~cm}^{-1}$ correspond to $\mathrm{O}-\mathrm{H}$ and $\mathrm{C}=\mathrm{O}$ stretching modes of $\mathrm{COOH}$, respectively, which are absent in the spectrum of the graphene sample. In addition, the absorption at $1553 \mathrm{~cm}^{-1}$ caused by the skeletal vibrations of graphene is observed.

thickness accurately. Fig. 3 shows the IR spectra of graphene 
oxide and graphene obtained by the hydrothermal reaction. In graphene oxide, a strong and broad absorption at $3440 \mathrm{~cm}^{-1}$ due to $\mathrm{O}-\mathrm{H}$ stretching vibration was seen. The $\mathrm{C}=\mathrm{O}$ stretching of the $\mathrm{COOH}$ groups situated at the edges of G-O platelets was observed at $1725 \mathrm{~cm}^{-1}$. The absorptions due to $\mathrm{O}-\mathrm{H}$ bending vibrations, epoxy groups, and skeletal ring vibrations were observed at around $1625 \mathrm{~cm}^{-1}$. The IR spectra of graphene confirmed the reduction of graphene oxide sheets. In the spectrum of graphene shown in Fig. 3, the absorptions at 3440, 1725 , and $1625 \mathrm{~cm}^{-1}$ were absent. However, a new absorption at around $1553 \mathrm{~cm}^{-1}$ was observed, this may be due to the skeletal vibrations of the graphene platelets [14-15].

We investigated the actuation of the bilayer papers as a function of the voltage at room temperature. Notably, the Ushaped beam started to deflect and its free end tilted up depending on the voltage (Fig. 4). There existed a threshold voltage varying with the structure of the bilayer paper samples. Under the threshold voltage, the variation of the beam can be ignored. We would pay more attention to the situation that the testing voltage was greater than the threshold voltage. At relative low voltage, the beam tilted towards the face of graphene oxide layer. With the increase of the voltage, the beam rolled up further and the curvature of the beam became larger and larger. In addition, the beam gradually unrolled and returned to the original shape (from the middle to the right in Fig. 4) when the power was turned off.

According to the previous literature [11], the actuation of the beams may be attributed to the internal heat generation caused by Joule heat. In order to verify the fact that the curling of the beam acted as a function of temperature, the actuators were inserted into a drying oven with a controllable set of temperature (Fig. 5). The beam started to curl as was expected when the temperature increased from $25^{\circ} \mathrm{C}$ to $75^{\circ} \mathrm{C}$. We also found that the beam started to unroll and finally returned to its original shape when the temperature decreased to $25{ }^{\circ} \mathrm{C}$. Generally, graphene layer possessed a layered structure as well as graphene oxide layer, however, graphene oxide sheets which had abundant oxygen-containing, functional groups differed from graphene sheets which owned limited functional groups. It was these functional groups that had great compacts on structure of graphene oxide layer and graphene layer from interaction among layers. Graphene papers had a good electrical conductance and smaller interlayer spacing, which caused a different mechanical properties and thermal

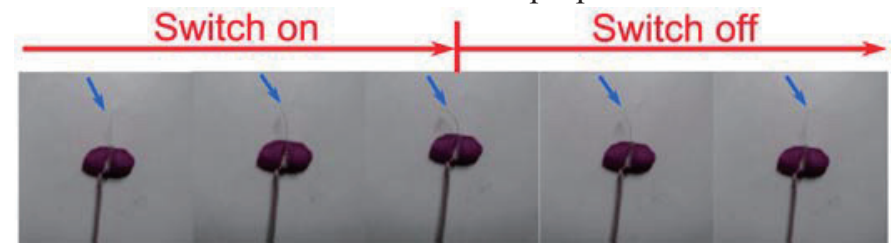

Fig. 4. The actuation of U-shaped beam caused by electrical voltage. When the power source was switch on, the beam began to deflect (from the left to the middle). The beam returned to its original state when the power source was switch off (from the middle to the right).

expansion coefficient compared to the graphene oxide papers. For U-shaped beams, graphene beams had a resistance with a few $K \Omega$, which would generated a great deal of heat when electric current passed through them. Consequently, the local temperature would rise, which would give birth to huge
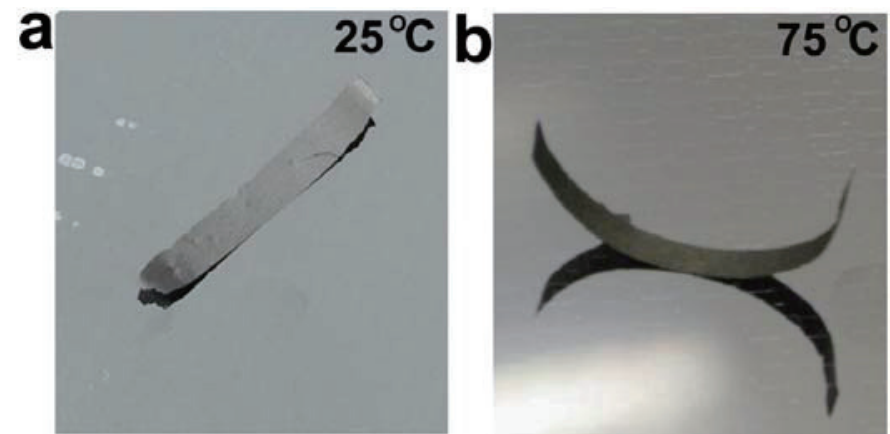

Fig. 5. The effect of the temperature on the deflection of the bilayer beam. When the temperature increased to $75^{\circ} \mathrm{C}$, the bilayer beam started to bend, and finally formed a semicircle. In addition, the bilayer beam returned back to its original state when the temperature decreased to $25^{\circ} \mathrm{C}$.

thermal stress in the interface between graphene layer and graphene oxide layer due to different thermal expansion coefficient. In addition, the elevation of temperature of graphene oxide layer was realized through heat transfer because the graphene oxide layer was electrical insulating, which did not generate heat during working. Thereby the temperature of graphene oxide layer would be slightly lower than that of graphene layer, which would increase the thermal stress resulting in large displacement. Accurately, thermal stress was the direct reason for large displacement.

In order to analyze the actuation of actuator in detail, we adopted a classical equation (1) depicted as follows [16]:

$$
\begin{gathered}
\frac{1}{r}=\frac{6 w_{1} w_{2} E_{1} E_{2} t_{1} t_{2}\left(t_{1}+t_{2}\right)\left(\alpha_{1}-\alpha_{2}\right) \Delta T}{\left(w_{1} E_{1} t_{1}^{2}\right)^{2}+\left(w_{2} E_{2} t_{2}^{2}\right)^{2}+2 w_{1} w_{2} E_{1} E_{2} t_{1} t_{2}\left(2 t_{1}^{2}+3 t_{1} t_{2}+2 t_{2}^{2}\right)} \\
\frac{1}{r}=\frac{6 E_{1} E_{2} t_{1} t_{2}\left(t_{1}+t_{2}\right)\left(\alpha_{1}-\alpha_{2}\right) \Delta T}{\left(E_{1} t_{1}^{2}\right)^{2}+\left(E_{2} t_{2}^{2}\right)^{2}+2 E_{1} E_{2} t_{1} t_{2}\left(2 t_{1}^{2}+3 t_{1} t_{2}+2 t_{2}^{2}\right)} \\
\frac{1}{r}=\frac{6 t_{1} t_{2}\left(t_{1}+t_{2}\right)\left(\alpha_{1}-\alpha_{2}\right) \Delta T}{t_{1}^{4}+t_{2}^{4}+2 t_{1} t_{2}\left(2 t_{1}^{2}+3 t_{1} t_{2}+2 t_{2}^{2}\right)}
\end{gathered}
$$

Where $t_{1}, w_{1}, \alpha_{1}, E_{1}$ are thickness, width, thermal expansion coefficient, and Young s modulus of graphene oxide layer, respectively. $t_{2}, w_{2}, \alpha_{2}, E_{2}$ represent thickness, width, thermal expansion coefficient, and Young's modulus of graphene layer, respectively. $l, r, \triangle T$, are on behalf of length, radius of curvature, difference between initial temperature and final temperature of macroactuator in sequence. The details are demonstrated in Fig. 6. For a given macroactuator, $l$ and $w$ are the same for both graphene paper and graphene oxide paper. Therefore, equation (1) can be simplified into (2). Apparently, the curvature of the beam is proportional to $\triangle T$. In addition, the greater difference between $\alpha_{1}$ and $\alpha_{2}$, the greater the change of the beam. According to the literature, both of graphene paper and graphene oxide paper own a nearly equal Young s modulus, which makes the (2) be reduced into the (3). 
It is noteworthy that the curvature of the beam can achieve a maximum when $E_{1}$ is equal to $E_{2}$. Consequently, the actuator based on graphene paper/graphene oxide paper can obtain greater displacement.

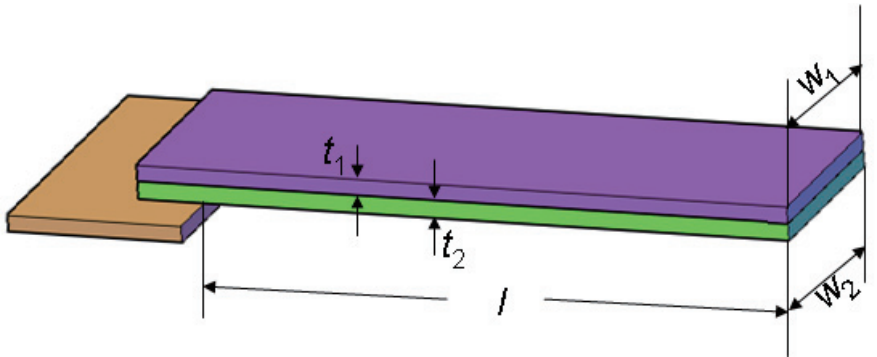

Fig. 6 The model of the beam based on graphene paper/graphene oxide paper.

Actuators based on graphene paper/graphene oxide paper can be applied to many fields. Taking artificial cilia for example, U-shaped beams can be assembled into arrays and all beams need to be divided into two groups A and B (Fig. 7a). Fig. 7 demonstrates the entire working process. In brief, at the beginning, all beams curled up to lift up object through turning on the voltage source (Fig. 7a). Then turn off the voltage source which was connected to the group B, which resulted in fall of beams in group B (Fig. 7b). In succession, the voltage source connected to group A was cut off, which decrease the displacement and move the object over a distance (Fig. 7c). Switching on the voltage source linked to group B made beams curl up again, which would lift up the object and move it over a distance further (Fig. 7d). Finally, the voltage source to the group A was switch on to make beams curl up (Fig. 7e) and the arrays came back to its initial state indicated by dash line. Compare Fig. 7 a with $7 \mathrm{e}$, it was clearly seen that the object moved some distance through one cycle. Moreover, to make a long move, many cycles are required to transport the object continuously.

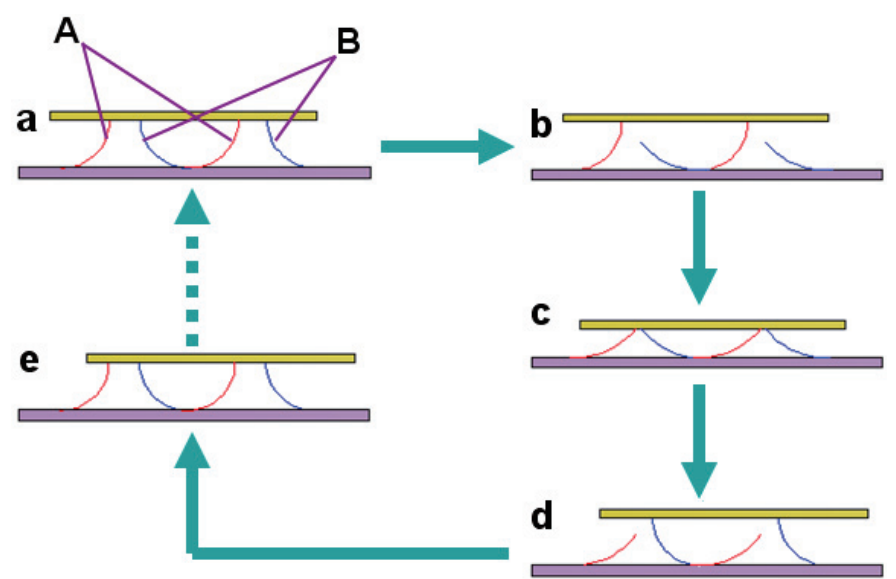

Fig. 7 The workflow of artificial cilia. The U-shaped beams can be assembled into arrays of cilia, which can be used to transport the objects.

\section{CONCLUSIONS}

A novel actuator based on graphene paper/graphene oxide papers has been fabricated through a facile method. The displacement of the actuators is changed linearly as a function of voltage. In addition, the thermal stress originating from the difference of the coefficient of thermal expansion of two kinds of papers is responsible for the bending of the beams. Similar Young's modulus for the two types of papers is in favor of great displacement. Actuators based on graphene/graphene oxide paper have many applications, e.g., transport of microchips.

\section{ACKNOWLEDGMENT}

This work was supported by the National Basic Research Program of China (Grant Nos. 2009CB623702 and 2011CB707601), and the National Natural Science Foundation of China (Nos. 61274114 and 51071044).

\section{REFERENCES}

[1] A.R. Ranjbartoreh, B. Wang, X.P. Shen, and G.X. Wang, "Advanced mechanical properties of graphene paper," J. Appl. Phys., vol. 109, pp. 014306-6, 2011

[2] O.C. Compton, and S.T. Nguyen, "Graphene oxide, highly reduced graphene oxide, and graphene: versatile building blocks for carbonbased materials," Small, vol. 6, pp. 711-723, 2010.

[3] D.A. Dikin, S. Stankovich, E.J. Zimney, R.D. Piner, G.H.B. Dommett, et al., "Preparation and characterization of graphene oxide paper," Nature, vol. 448, pp. 457-460, 2009.

[4] C.Y. Li, T.W. Chou, "Electrostatic charge distribution on single-walled carbon nanotubes,” Appl. Phys. Lett., vol. 89, pp. 063103, 2006.

[5] D.J. Jones, S.E. Prasad, J.B. Wallace, "Piezoelectric materials and their applications: key engineering materials," Adv. Ceram. Mater., vol. 122124, pp. 71-143, 1996.

[6] T. Rueckes, K. Kim, E. Joselevich, G.Y. Tseng, C.L. Cheung, et al., "Carbon nanotube-based nonvolatile random access memory for molecular computing,” Science, vol. 289, pp. 94-97, 2000.

[7] J.E. Jang, S.N. Cha, Y. Choi, G.A.J. Amaratunga, D.J. Kang, et al., "Nanoelectromechanical switches with vertically aligned carbon nanotubes," Appl. Phys. Lett., vol. 87, pp. 163114, 2005.

[8] L. Forró, "Nanotechnology: beyond gedanken experiments," Science, vol. 289, pp. 560-561, 2000.

[9] V.V. Deshpande, H.Y. Chiu, H.W.C. Postma, C. Miko, L. Forró, et al., "Carbon nanotube linear bearing nanoswitches," Nano Lett., vol. 6, pp. 1092-1095, 2006.

[10] S.E. Zhu, R. Shabani, J. Rho, Y. Kim, B.H. Hong, et al., "Graphenebased bimorph microactuators," Nano Lett., vol. 11, pp. 977-981, 2011.

[11] S.J. Park, J.H. An, J.W. Suk, and R.S. Ruoff, "Graphene-based actuators," Small, vol. 6, pp. 210-212, 2009.

[12] Q.Z. Liang, X.X. Yao, W. Wang, Y. Liu, and C.P. Wong, "A threedimensional vertically aligned functionalized multilayer graphene architecture: an approach for graphene-based thermal interfacial materials," ACS Nano, vol. 5, pp. 2392-2401, 2011.

[13] W.S. Hummers, R.E. Offeman, "Preparation of graphitic oxide," J. Am. Chem. Soc., vol. 80, pp. 1339, 1958.

[14] G. Wang, B. Wang, J. Park, J. Yang, X. Shen, et al., "Synthesis of enhanced hydrophilic and hydrophobic graphene oxide nanosheets by a solvothermal method," Carbon, vol. 47, pp. 68-72, 2009.

[15] A.V. Murugan, T. Muraliganth, and A. Manthiram, "Rapid, facile microwave-solvothermal synthesis of graphene nanosheets and their polyaniline nanocomposites for energy strorage," Chem. Mater., vol. 21, pp. 5004-5006, 2009.

[16] C. Liu, Foundations of MEMS. China Machine Press, 2007. 